\section{CPS-115 RITUXIMAB IN THE TREATMENT OF PRIMARY GLOMERULONEPHRITIS}

M Lamas Lopez*, C Orallo Luna, J Fra Yañez, L Becerra Injerto, A Fernández González, N González Sánchez, P Puente Martínez. Hospital Universitario San Agustín, Servicio de Farmacia Hospitalaria, Avilés, Spain

10.1136/ejhpharm-2019-eahpconf.264

Background Primary glomerulonephritis is usually treated with steroids and immunosuppressants, however, some patients exhibit treatment resistance or severe toxicity related to the chronic treatment. It has been observed that the use of offlabel rituximab could be an effective alternative to be used as treatment.

Purpose To assess the efficacy and safety of rituximab in the treatment of primary glomerular disease.

Material and methods Observational, retrospective study of all patients with primary glomerulonephritis treated with rituximab between 2012 and 2017. For data collection, the eletronic clinic history system (Selene) was used, as was SPSS-Stadistics for the statistical analysis. Data registered: sex, age, histological classification, dose and posology of rituximab. Efficacy was assessed comparing urine protein in a 24 hour period (UP-24), serum albumin (Alb), serum creatinine $(\mathrm{Cr})$ and treatments with corticosteroid and immunosuppressants before and 6 months after treatment with rituximab. The profile of adverse reactions was recorded to assess safety.

Results Thirty-six patients (19 male and 17 female) were included with an average age of $60.32 \pm 14.94$ years and histological diagnosis of membranous nephropathy (61.2\%), focal segmental glomerulosclerosis (16.6\%), minimal change disease $(11.1 \%)$ and membranoproliferative glomerulonephritis (11.1\%). The dosing regimens were two doses of $1 \mathrm{~g}$ of rituximab separated by 15 days $(72.2 \%)$ and one single dose of $1 \mathrm{~g}(27.8 \%)$.

Six months after the beginning of treatment, the mean UP-24 (range 0.04-0.15 g/24 hour) decreased from 2.87 $\pm 2.46 \mathrm{~g} / 24$ hour to $1.09 \pm 0.77 \mathrm{~g} / 24$ hour $(\mathrm{p}=0,001)$ normalising in $8.3 \%$ of patients. The mean Alb (range $3.5-5.2 \mathrm{~g} / \mathrm{dL}$ ) increased significantly from $3.3 \pm 0.7 \mathrm{~g} / \mathrm{dL}$ to $3.9 \pm 0.5 \mathrm{~g} / \mathrm{dL}$ $(\mathrm{p}<0.005)$ and the mean $\mathrm{Cr}$ (range $0.7-1.20 \mathrm{mg} / \mathrm{dL}$ ) decreased from $1.24 \pm 0.56 \mathrm{mg} / \mathrm{dL}$ to $1.21 \pm 0.55 \mathrm{mg} / \mathrm{dL}$ $(\mathrm{p}=0.436)$.

Twelve patients used corticosteroids, of which 75\% were able to discontinue them and $25 \%$ decreased the dose. Twenty-three patients used immunosuppressants, of which $78.2 \%$ could be discontinued and $21.8 \%$ reduced the dose.

Sixteen per centof patients had some adverse reaction, all related to perfusion (skin rash, sore throat and pruritus).

Conclusion Rituximab is an effective alternative for the treatment of primary glomerulonephritis. It significantly improves levels of UP and Alb, as well as allowing the suspension or reduction of doses of corticosteroid and immunosuppressant treatments with an acceptable profile of adverse reactions, all related to the administration of the drug.

\section{REFERENCES AND/OR ACKNOWLEDGEMENTS}

No conflict of interest.

\section{CPS-116 VEMURAFENIB-INDUCED STEVENS-JOHNSON SYNDROME IN A PATIENT WITH METASTATIC MELANOMA: A CASE REPORT}

${ }^{1} \mathrm{M}$ Lavandeira Pérez*, ${ }^{1} \mathrm{P}$ de Juan-García Torres, ${ }^{2} \mathrm{~F}$ Fernández-Rañada, ${ }^{3} \mathrm{MA}$ Vega Castro, ${ }^{1}$ AM Horta Hernández. ${ }^{1}$ Hospital Universitario de Guadalajara, Hospital Pharmacy, Guadalajara, Spain; ${ }^{2}$ Hospital Universitario de Guadalajara, Medical Oncology, Guadalajara, Spain; ${ }^{3}$ Hospital Universitario de Guadalajara, Allergology, Guadalajara, Spain

\subsection{6/ejhpharm-2019-eahpconf.265}

Background Vemurafenib and dabrafenib are BRAF inhibitors used for the treatment of unresectable or metastatic melanoma (MM) with BRAF V600 mutation. Stevens-Johnson syndrome (SJS) has been rarely reported with vemurafenib and is not described with dabrafenib. Severe adverse reactions have been described in vemurafenib-treated patients who had previously received nivolumab.

Purpose To describe a severe case of vemurafenib-induced SJS in a patient with $\mathrm{MM}$ previously treated with nivolumab.

Material and methods This was a descriptive and retrospective clinical case. Data were obtained by review of electronic medical records.

Results A 67-year-old woman was diagnosed with vulvar melanoma Clark level III, Breslow thickness of $0.8 \mathrm{~mm}$. She initially underwent surgery in October 2007.

In May 2016, pulmonary nodules and local recurrence were detected and BRAF mutation was positive. She received nine cycles of nivolumab from August 2016. In January 2017, disease progression was observed and second-line treatment with vemurafenib-cobimetinib was started. Nine days after beginning this treatment, a severe cutaneous reaction appeared. The Dermatology and Allergy Departments diagnosed it as a SJS. The Naranjo Algorithm established as 'probable' (score 4) the relationship between vemurafenib and SJS. Dabrafenib was evaluated as an alternative treatment in a clinical session with the Allergy, Oncology and Pharmacy Departments. Cross-reactivity between vemurafenib and dabrafenib has been described. This led to the performance of an in vitro lymphocyte transformation test (LTT) assay with both BRAF inhibitors. Results were positive for vemurafenib and negative for dabrafenib and sulfametoxazol (control). Treatment with dabrafenib was started in July 2017 in the Allergy Outpatient Clinic with good tolerance and without skin reactions. The patient died in December 2017 after disease progression.

Conclusion

- Previous treatment with nivolumab could worsen vemurafenib safety profile as described in several case reports.

- A negative LTT cannot discard cross-reactivity between BRAF inhibitors, but it might lead to careful administration of dabrafenib as an alternative therapy.

- Multidisciplinary approach is key in treatment decisions due to hypersensitivity reactions.

\section{REFERENCE AND/OR ACKNOWLEDGEMENTS}

1. Bellón $T$, Lerma $V$, González-Valle 0 , et al. Vemurafenib-induced toxic epidermal necrolysis: possible cross-reactivity with other sulfonamide compounds. $\mathrm{Br} J \mathrm{Der}$ matol 2016;174:621-4.

No conflict of interest. 